Clarke R, Heath G, Pattison H, Farrow C. (2008). Weight-management in children living with asthma: a qualitative study of the experiences of paediatric healthcare professionals. Journal of Asthma, 16:1-8. doi: 10.1080/02770903.2018.1536146.

\title{
Weight-management in children living with asthma: A qualitative study of the experiences of paediatric healthcare professionals
}

\author{
Rebecca Clarke $^{1}$, Gemma Heath ${ }^{1,2}$, Helen Pattison ${ }^{1}$, Claire Farrow ${ }^{1 *}$ \\ ${ }^{1}$ Department of Psychology, Aston University, Birmingham, UK \\ ${ }^{2}$ Department of Psychology, Birmingham Children's Hospital, Birmingham, UK \\ *c.farrow@aston.ac.uk, 01212045384
}

\begin{abstract}
:
Objective: Weight loss has been found to improve the symptoms of asthma in children who are overweight. However, many paediatric weight management programmes do not address the challenges associated with living with asthma. The aim of this study was to explore the views and experiences of paediatric healthcare professionals concerning weight management advice and support offered to families of children living with asthma. Methods: In-depth individual interviews with 10 healthcare professionals who work with a paediatric asthma population ( $n=4$ Respiratory Consultants, 3 Respiratory Nurses, 3 General Paediatricians). Data were analysed using a Framework approach. Results: Healthcare professionals highlighted that families' perceptions of weight, their approach to physical activity and nutrition, the family's social context and perceptions of asthma and asthma treatment all influence weight management in children living with asthma. Initiating weight management conversations and referring to weight management support were perceived as challenging. It was thought that tailoring weight management to the needs of children living with asthma and locating support within the community were important to the success of a family-centred intervention. Conclusion: The results highlight the added complexity of responding to excessive weight in a paediatric population with asthma. Training and referral guidance for healthcare professionals may help overcome weight management support challenges. Addressing family beliefs about the factors influencing paediatric asthma and exploring families' motivations for behaviour change may enhance engagement with weight management.
\end{abstract}


Keywords: Obesity, respiratory, adolescence, management, health services

\section{Introduction}

Asthma is one of the most common chronic illnesses in children. Evidence suggests that children living with asthma have an increased risk of developing obesity $(1,2)$. Maintaining a healthy weight is of particular importance in paediatric asthma. Obesity in children living with asthma can result in increased inflammation, higher levels of hospitalisation and three times greater use of medication compared to children living with asthma who have a healthy weight (3-4). To date, the mechanisms underlying the relationship between asthma and obesity are not clear. Some theories propose that obesity precedes asthma as increased adiposity can lead to airway obstruction and increased systematic inflammation (5). Other research suggests that the challenges posed by living with asthma contribute to an increased risk of obesity as lifestyle factors, such as reduced physical activity levels, are thought to mediate the relationship between asthma and obesity (6).

The US National Asthma Education and Prevention Program recommends weight management to improve overall health and asthma control in both adults and children (7). Yet most paediatric weight management programmes are not tailored to the unique needs of living with asthma, such as addressing parental anxieties that arise from breathlessness when exercising and the child's perceptions of exercise parameters $(8,9)$. So far, the limited weight management programmes designed specifically for children living with asthma have shown promising results in improving asthma control and reducing weight (10). However, little is known about the best ways to provide weight management support for paediatric asthma population as there are no current clinical guidelines on how to do so (11).

Doctors, nurses and other healthcare professionals (HCPs) play a key role in providing weight management support. Currently, weight management is promoted by the UK National Health Service (NHS) initiative 'Make Every Contact Count'. This initiative encourages HCPs to provide opportunistic health behaviour change advice during every patient contact. Alongside this, strategies have been developed to help healthcare professionals manage weight, such as recommendations to learn local weight management pathways and to how correctly determine children's BMI percentile (12). 
Despite guidelines on how to support healthy weight maintenance in clinical practice, several barriers have been identified to hinder support provision. HCPs, such as junior doctors, nurses and allied health professionals, have reported that they do not perceive it as within their job role, have limited resources to do so and feel uncomfortable discussing patient weight $(13,14)$. There is also an added sensitivity to discussing child weight due to the stigma attached to overweight and obesity (15). Literature suggests that doctors and nurses worry that parents will react defensively to child weight being raised in clinic which could compromise the patientphysician relationship $(14,16)$.

In order to optimise the weight management support provided for paediatric asthma populations, it is important to understand how weight is managed currently and what changes could be made to improve support and advice. The purpose of this study was to explore the views and experiences of healthcare professionals concerning the weight management advice and support offered to families' and children living with asthma.

\section{Method}

\section{Recruitment}

Healthcare Professionals (doctors and nurses) providing secondary and tertiary care to children and young people with a clinical asthma diagnosis were identified at a specialist Children's hospital and sent an invitation letter and information sheet about the study via email. Interested HCPs then contacted the researchers directly via email or telephone to receive more information about the study and to arrange a time/place for a one-to-one interview. Purposive sampling was used to recruit HCPs with a variety of perspectives and experiences. Recruitment commenced following a favourable ethical opinion from a local University ethics committee (Project \#995).

\section{Data collection}

A qualitative approach was used to ensure an in-depth narrative could be collected. The interviews followed a semi-structured approach to allow for flexibility. Interview schedules were developed in accordance with 
research literature and the study objectives. Questions focused on experiences of working with children with asthma-obesity comorbidity, managing weight in this population and the barriers and facilitators of providing support. Before interviews took place, informed consent and demographic data were obtained from participants. Interviews averaged 45 minutes and were conducted by a member of the research team. Each participant received a $£ 10$ voucher to thank them for their time.

Data analysis

All interviews were audio-recorded, transcribed verbatim and anonymised. Data were analysed using the Framework method (17). Two researchers followed a systematic approach of familiarisation with the data and independent coding to highlight key concepts. Consensus on coding was reached through discussion with a third researcher. Implicit connections between the codes led to the formation of categories and a thematic framework, which was refined over subsequent readings of the transcripts and applied to the whole data set. Data were then summarised and entered into a matrix devised from the thematic framework. This process facilitated the comparison of participants' views by case and code allowing for commonalities and differences to be identified. Finally, interpretive themes were generated from the categorised data, describing patterns, relationships and concepts related to weight management in children living with asthma. Data management and retrieval was assisted by the use of NVivo software V11.

\section{$\underline{\text { Results }}$}

In total, 10 Healthcare Professionals participated (Table 1). Three core themes were generated through data analysis: (1) factors affecting health behaviours, (2) current weight management support in practice and (3) developing weight management interventions. While all participants described a relationship between asthma and obesity, a common thread running through the data was professional uncertainty regarding the direction of this relationship. In attempting to understand the association between poor asthma control and increased weight, several vicious cycles were identified. This thread was evident throughout the three main themes and is thus not reported separately.

Factors affecting health behaviours. 
Professionals suggested that parental anxiety around exercise parameters was "usually the main reason why children are inactive" (General Paediatrician 3) and that this anxiety could be transferred from parent to child, which may be difficult to mitigate in later life. Misconstruing exercise intolerance for asthma symptoms was thought by HCPs to reduce confidence in a child's exercise parameters, lead to avoidance behaviours and a "special get-out-of jail-free card" (General Paediatrician 3) and a decline in physical activity engagement. It is here that the first viscous cycle of difficulty in exercising, weight gain and a decline in asthma control is alluded to. HCPs thought that low activity levels at school stemmed from family and child concerns around how some teachers responded to children's breathlessness: “some PE teachers have said that they are actually making it up to get out of exercise" (Respiratory Consultant 4).

Similarly, HCPs recognised that the use of some asthma medications (e.g. steroids) could result in increased appetite and weight gain and reported families' complaints about this. Treatment doses and weight were checked regularly to avoid unnecessary steroid-induced weight gain as "managing the obesity does become a primary aim of their treatment" (Respiratory Consultant 1). This contrasted with a nurse who stated that children using steroids "haven't got a choice, they're just going to be heavier" (Respiratory Nurse 3). Another HCP felt "forced" (Respiratory Consultant 2) to give children steroids because of poor medication adherence, stemming from a perception that inhalers do not constitute 'real medicine'. It may be that some HCPs accept weight gain from steroids as a necessity to asthma control. Yet many professionals reported concerns of a vicious cycle in steroid use. It was thought that poor asthma control required steroids for which resulting weight gain made exercise more challenging. As a consequence, exacerbated asthma symptoms then required the need for more steroids.

Changes in society such as a rise in obesity prevalence were also thought to be influential. It was suggested that parental perceptions of obesity as normative and the view that it is possible to be overweight and healthy posed a challenge to changing beliefs about the medical consequences of obesity. It was thought that parents were less concerned about children being overweight. In contrast, HCPs compared obesity to negative health behaviours such as smoking, implying that responsibility for weight management was interpreted differently between families and HCPs: 
"it's been judged that that's not appropriate for children to make the choice to smoke because the health consequences are very bad and, for some reason, [weight] is not viewed in the same way" (General Paediatrician 3).

Furthermore, food was perceived by HCPs to be used as a tool by parents to modify child behaviour, rather than simply providing a source of nourishment. One example was given, when a parent gave their child crisps in a consultation "to keep the child relatively quiet" (Respiratory consultant 3). As well as parents utilising food, HCPs felt that young people themselves turned to food as a way to cope with having asthma: "they get all sad and then some people turn to food" (Respiratory Nurse 3). Mindless eating and eating through boredom were suggested as ways of coping with the perceived restrictions in physical activity imposed by asthma.

\section{Weight management support in practice.}

Healthcare professionals felt that initiating conversations about a child's weight were difficult: “it's the elephant in the room" (Respiratory Nurse 2). While having a strong rapport with families helped HCPs to feel more comfortable discussing weight, the desire to maintain relationships added further pressure. Participants expressed concern at pursuing weight management conversations if it would harm the physician-patient relationship. Professionals recognised the importance of addressing the topic, but in a case where a patient struggled with difficult-to-manage asthma, one respiratory nurse worried about causing immediate distress: "Another stick to beat them with, but then, it's hard because you know, we all know that we should [because] the outcomes have improved" (Respiratory Nurse 2). It was felt by some professionals that they were "illprepared" for starting weight management conversations (Respiratory Consultant 4). Information sharing with experienced clinicians who had developed these skills or training which included families' input were suggested to help.

Medicalising the initiation of weight management conversations with growth charts was said to help conversations, with medical statistics conceived as easy to refer to when emphasising the importance of maintaining a healthy weight. One General Paediatrician explained by doing so "the personalities are taken out of the equation" (General Paediatrician 2). However, a lack of evidence that losing weight could improve asthma was considered to make promoting weight loss more difficult. It was implied that the possibility of asthma improvement for patients isn't a sufficient incentive to outweigh the difficulty of trying to lose weight. 
Where possible, professionals referred families to dietetics, psychology and school nurses, as well as referring back to the patients' GP for weight management support. Yet, participants expressed that they were "not certain that GPs are the right people to refer to" (Respiratory Consultant 1) indicating a lack of certainty about weight management support protocols. Professionals desired more dialogue with other HCPs following referrals as they received no feedback on families' engagement and had no way to monitor the patients' weight between asthma consultations. A sense of feeling "powerless" arose (Respiratory Consultant 3) over limitations in the availability of resources as many weight management programmes had been decommissioned: "it is like making a diagnosis and saying, 'Well I can't actually help you'” (Respiratory Consultant 4). Professionals sought somewhere to refer families to for weight management support before the complications associated with obesity began: "So [unless] they've hit the bottom, they don't receive any service. Down there, there is no parachute" (General Paediatrician 1).

Opinion on whose responsibility it was to manage weight varied. Some professionals felt they had "a responsibility not to ignore" (Respiratory Consultant 3) weight concerns and were able to provide brief, opportunistic interventions. However, some questioned whether they were the right person to monitor weight long-term due to capacity and expertise. It was suggested that community based HCPs were better placed to monitor weight and provide health promotion. There was a sense that weight management should be more than just a responsibility for medical professionals, but that it should be integrated into public health and that greater emphasis should be placed on prevention. One Respiratory Consultant explained that although support was provided for families they had to "be very clear that this is their [the families] responsibility still" (Respiratory Consultant 1) and identified that any success was down to them as the "hard work is done with the family" (General Paediatrician 3). HCPs perceived that some families did not take responsibility for a child's weight, instead attributing responsibility to factors 'beyond' their control, such as genetics or disease.

\section{Developing weight management interventions.}

Healthcare professionals recommended tailoring generic weight management to suit the needs of an asthma population. Educational components that they would like included comprised: education on diet, such as calorie intake, portion size, healthy food alternatives that accommodate for different cultural backgrounds and eating with the family. Alongside this, it was suggested that specialised exercise education should be included, such as 
the "recognition that asthma does come with exercise related symptoms" (General Paediatrician 1). Education on the health consequences of obesity was recognised as important to increase awareness. In addition, the majority of HCPs felt that psychological interventions could support patients with the pressures of living with asthma, adherence to asthma treatment and weight management and with changing parental attitudes and behaviour. Including peer support was also suggested so that children and families feel less isolated and are able to see that progress is attainable.

Healthcare professionals felt that it was important that education and support was provided for the whole family as opposed to the child in isolation. A General Paediatrician explained that families with successful weight loss reported that they were only successful because the family "wholeheartedly signed up" (General Paediatrician 1). Only one participant suggested that families may not need to be involved in a weight management programme as it can be "addressed by actually educating and supporting the professionals looking after them" (Respiratory Consultant 4).

One General Paediatrician felt that it may be important to have a varied weight range included as prevention was also important and including only overweight children may normalise overweight and reduce motivation to change. Whilst most HCPs felt an intervention should be aimed at any age, two General Paediatricians believed that early interventions to target obesity were required to prevent excess weight before it is engrained and behaviour is more difficult to change.

For HCPs, family engagement and encouraging attendance to additional healthcare appointments when families already take time off school and work for their asthma care was thought to be challenging. To increase longterm engagement in weight maintenance, HCPs suggested finding a unique way to "hook" patients in; to "tease out and draw out what it is that would be the motivator" (General Paediatrician 1) and incorporating a variety of activities and technology to promote engagement.

Professionals stressed that a weight management programme could be led by anyone with a good knowledge of asthma. However, a multi-disciplinary approach with involvement from school nurses, community nurses, dieticians, physiotherapists, psychologists and school teachers was suggested as the basis of a successful weight management team. Participants reported that programmes should happen over a period of time, facilitating 
patients to build trust and rapport with programme leaders. To ensure attendance, it was felt important for any programme to be as convenient as possible for families. Running interventions outside of school time, having information accessible out of hours and moving weight management support into the patients' community were suggested as ways to do this so that the change wouldn't be "disrupting the normal life of that child and the family" (Respiratory Consultant 1). Several HCPs suggested that school would be a good environment for providing weight management support as "all children go to school" and "parents have to interact with school" (General Paediatrician 3).

\section{$\underline{\text { Discussion }}$}

The current study explored healthcare professionals' views and experiences of weight management and the barriers and facilitators to providing support for a paediatric asthma population. The results of this study illuminate healthcare professionals' uncertainty and differing views regarding the nature of the asthma-obesity relationship. This could have implications for how professionals approach weight management in patients with asthma and may consequently lead to different health outcomes for children. Several vicious cycles were identified throughout this study that highlight how living with asthma can create challenges to weight management and lead to increased asthma symptoms and weight gain. Physical activity, eating behaviour, family influences and asthma medication were all recognised as influencing weight management in this paediatric asthma population.

HCPs acknowledged that asthma severity could restrict physical activity levels. However, participants believed that breathlessness during exercise was often misinterpreted as a symptom of asthma by parents and children rather than an indication of fitness. Perceiving breathlessness as a symptom of asthma was thought to decrease children's confidence in their exercise parameters. The outcome of this misinterpretation may be that children restrict their activity levels and subsequently have lower fitness ability and develop exercise avoidance behaviours $(18,19)$. As has been previously documented in the literature, participants in this study suggested that restricting exercise may also develop from fear of exercise-induced asthma attacks (8). Research has shown that parental anxiety can lead to children developing avoidance behaviours in asthma-threat scenarios (20). This finding was also supported by the health professionals interviewed here. It could be that both children and parents require further education to correctly attribute breathlessness to either asthma or fitness ability, thus 
working to prevent cycles of exercise avoidance, increased weight, lower fitness ability and worse asthma symptoms, as well as preventing the transfer of anxiety from parent to child.

Participants recognised that parents used food as a tool to influence child behaviour. The use of food as a reward and controlling feeding practices are known to impact on child eating behaviour and can influence eating and weight gain $(21,22)$. HCPs discussed how older children used food as a way of coping with living with asthma, echoing research findings that adolescents living with asthma are more likely to have unhealthy eating behaviours (23). However, it is possible that children living with asthma may overeat when bored or due to perceived lower exercise parameters $(9,18)$. For example, research has found that salty-snack consumption and television watching are associated with increased asthma symptoms (24). Diets high in salt and fat have been linked to increased airway inflammation and wheezing $(24,25)$. Such symptoms are likely to decrease exercise tolerance and make weight management engagement more difficult, reflecting HCPs suggestions of vicious cycles furthering weight gain and reduced asthma control.

Healthcare Professionals reported parental concern around the use of steroids for asthma treatment leading to increased appetite and weight gain. It is possible that this worry could result in poor medication adherence linking to previous research which has found that parental medication concerns are associated with low medication adherence in children living with asthma (26). Likewise, research indicates that concerns about medication-induced weight gain can create a desire to avoid steroid use in adolescent asthma patients (27). In their Necessity and Concerns Framework, Horne and Weinman (28), suggest that concerns around medication can often outweigh the perceived need to take medication. HCPs described reviewing patient weight and medication regularly to avoid unnecessary weight gain, yet, some HCPs felt that the use of steroids was necessary for asthma control. As overweight and obese children have a reduced response to steroid treatment, a continued emphasis should be placed on weight management to improve both asthma control and treatment adherence in families and children living with asthma (29).

Additional paediatric asthma weight management obstacles were identified at the HCP level. Consistent with previous research, stigma associated with discussing child weight was recognised, with communication around weight perceived as uncomfortable for professionals, even when referring to medical statistics or having a good rapport with patients $(13,15)$. It was thought that a strong therapeutic relationship helped to aid conversations, 
though as previously reported, HCPs expressed concern that addressing weight with a child and their family may jeopardise this relationship and that parents would react negatively $(16,30)$. Contrary to this fear however, previous studies indicate that parents and adolescents are often supportive of HCPs addressing, discussing and monitoring adolescent weight (31). Other barriers to providing support included limited resources and a poor awareness (or existence) of referral pathways. Frustration with this lack of support services mirrored an overweight adolescent asthma sample who expressed unhappiness at weight concerns being raised in consultations without HCPs being able to provide further intervention (32). In addition, the hospital-based HCPs in this study felt that delivery of behaviour change support was better placed within community care settings, as found previously in other specialised HCPs (33). Clearer guidelines on weight management support and improved local referral pathways could help bridge the gap across different healthcare sectors.

Finally, professionals in this study discussed how any new weight management programme would need to be tailored to the needs of an asthma population and incorporate education on nutrition, exercise and obesity. It was recognised that psychological support was required to help young people manage living with asthma and adhere to both asthma and weight management. It is important that weight management advice used in practice is acceptable to patients and families to enhance engagement. Complementing the views of parents and adolescents $(34,35)$, HCPs believed that involving the child's family, enhancing motivation and ongoing support in the community would help to sustain engagement with weight management..

\section{$\underline{\text { Limitations }}$}

Although the purposive sample recruited a variety of job roles to capture different views and experiences, the participants for this study came from one specialist Children's Hospital. As a result, the views and experiences may only reflect that of the local area and those of HCPs working with a severe paediatric asthma population. Recruiting a larger sample from a range of hospitals may be useful in future research, as different training and different local weight management programmes may influence the weight management support offered. However, whilst not generalizable to the population at large, findings from this study are likely to be transferable to other paediatric overweight populations in the UK.

\section{Conclusion}


In conclusion, the results of this study highlight the complexity and variety of influences on managing weight in a paediatric asthma population. Tailoring family-centred weight management to the needs of a paediatric asthma population and locating support in to the community were thought to be key to the effectiveness and uptake of an intervention. Providing training on sensitive conversation initiation and guidance on referral pathways may reduce the uncertainty and discomfort of providing weight management support in clinical practice, as well as encouraging more dialogue between multi-disciplinary healthcare professionals to promote ongoing support. Providing education to families alone isn't enough to support weight behaviour (36). Future research would benefit from understanding families' views and experiences of the barriers and facilitators to paediatric weight management in asthma, alongside motivation to engage in weight management.

\section{$\underline{\text { References }}$}

1. Chen ZH, Salam MT, Alderete TL, Habre R, Bastain TM, Berhane K, et al. Effects of Childhood Asthma on the Development of Obesity among School-aged Children. American Journal of Respiratory and Critical Care Medicine. 2017;195(9):1181-8. doi: 10.1164/rccm.201608-1691OC. PubMed PMID: WOS:000400258300027.

2. Azizpour Y, Delpisheh A, Montazeri Z, Sayehmiri K, Darabi B. Effect of childhood BMI on asthma: a systematic review and meta-analysis of case-control studies. Bmc Pediatrics. 2018;18. doi: 10.1186/s12887018-1093-z. PubMed PMID: WOS:000430933600001.

3. Michelson PH, Williams LW, Benjamin DK, Barnato AE. Obesity, inflammation, and asthma severity in childhood: data from the National Health and Nutrition Examination Survey 2001-2004. Annals of Allergy Asthma \& Immunology. 2009;103(5):381-5. PubMed PMID: WOS:000271913400005.

4. Mosen DM, Schatz M, Magid DJ, Carnargo CA. The relationship between obesity and asthma severity and control in adults. Journal of Allergy and Clinical Immunology. 2008;122(3):507-11. doi: 10.1016/j.jaci.2008.06.024. PubMed PMID: WOS:000259234000010.

5. Shore SA. Obesity and asthma: Possible mechanisms. Journal of Allergy and Clinical Immunology. 2008;121(5):1087-93. doi: 10.1016/j.jaci.2008.03.004. PubMed PMID: WOS:000255961700002.

6. Leinaar E, Alamian A, Wang L. A systematic review of the relationship between asthma, overweight, and the effects of physical activity in youth. Annals of Epidemiology. 2016;26(7):504-10. doi: 10.1016/j.annepidem.2016.06.002. PubMed PMID: WOS:000380866600010. 
7. National asthma education and prevention program - Expert panel report 3 (EPR-3): Guidelines for the diagnosis and management of asthma - Summary report 2007. Journal of Allergy and Clinical Immunology. 2007;120(5):S94-S138. doi: 10.1016/j.jaci.2007.09.029. PubMed PMID: WOS:000251247600002.

8. Kornblit A, Cain A, Bauman LJ, Brown NM, Reznik M. Parental Perspectives of Barriers to Physical Activity in Urban Schoolchildren With Asthma. Academic Pediatrics. 2018;18(3):310-6. PubMed PMID: WOS:000429629200012.

9. Pianosi PT, Davis HS. Determinants of physical fitness in children with asthma. Pediatrics. 2004;113(3):E225-E9. doi: 10.1542/peds.113.3.e225. PubMed PMID: WOS:000189344400056.

10. Jensen ME, Gibson PG, Collins CE, Hilton JM, Wood LG. Diet-induced weight loss in obese children with asthma: a randomized controlled trial. Clinical and Experimental Allergy. 2013;43(7):775-84. doi: 10.1111/cea.12115. PubMed PMID: WOS:000320781100011.

11. Bateman ED, Hurd SS, Barnes PJ, Bousquet J, Drazen JM, FitzGerald JM, et al. Global strategy for asthma management and prevention: GINA executive summary (vol 31,pg 143, 2008). European Respiratory Journal. 2018;51(2). doi: 10.1183/13993003.51387-2007. PubMed PMID: WOS:000424396500001.

12. National Institute for Health and Care Excellence. Obesity in children and young people: prevention and lifestyle weight management programmes. NICE [Internet]. 2015 July [cited 2018 Jun 5]. Available from: https://www.nice.org.uk/guidance/qs94

13. Elwell L, Powell J, Wordsworth S, Cummins C. Health professional perspectives on lifestyle behaviour change in the paediatric hospital setting: a qualitative study. Bmc Pediatrics. 2014;14. doi: 10.1186/1471-243114-71. PubMed PMID: WOS:000335411800001.

14. Steele RG, Wu YP, Jensen CD, Pankey S, Davis AM, Aylward BS. School Nurses' Perceived Barriers to Discussing Weight With Children and Their Families: A Qualitative Approach. Journal of School Health. 2011;81(3):128-37. doi: 10.1111/j.1746-1561.2010.00571.x. PubMed PMID: WOS:000287492200002.

15. Puhl RM, Heuer CA. Obesity Stigma: Important Considerations for Public Health. American Journal of Public Health. 2010;100(6):1019-28. doi: 10.2105/ajph.2009.159491. PubMed PMID: WOS:000277722500020. 16. Walker O, Strong M, Atchinson R, Saunders J, Abbott J. A qualitative study of primary care clinicians' views of treating childhood obesity. Bmc Family Practice. 2007;8:7. doi: 10.1186/1471-2296-8-50. PubMed PMID: WOS:000250056400001. 
17. Gale NK, Heath G, Cameron E, Rashid S, Redwood S. Using the framework method for the analysis of qualitative data in multi-disciplinary health research. Bmc Medical Research Methodology. 2013;13. doi: 10.1186/1471-2288-13-117. PubMed PMID: WOS:000324780100001.

18. Kitsantas A, Zimmerman BJ. Self-efficacy, activity participation, and physical fitness of asthmatic and nonasthmatic adolescent girls. Journal of Asthma. 2000;37(2):163-74. doi: 10.3109/02770900009055439. PubMed PMID: WOS:000086701900006.

19. Park SJ, Sawyer SM, Glaun DE. Childhood asthma complicated by anxiety: An application of cognitive behavioural therapy. Journal of Paediatrics and Child Health. 1996;32(2):183-7. doi: 10.1111/j.14401754.1996.tb00919.x. PubMed PMID: WOS:A1996UL94400020.

20. Sicouri G, Sharpe L, Hudson JL, Dudeney J, Jaffe A, Selvadurai H, et al. Threat interpretation and parental influences for children with asthma and anxiety. Behaviour Research and Therapy. 2017;89:14-23. doi: 10.1016/j.brat.2016.11.004. PubMed PMID: WOS:000392779600002.

21. Farrow CV, Haycraft E, Blisset JM. Teaching our children when to eat: how parental feeding practices inform the development of emotional eating-a longitudinal experimental design. American Journal of Clinical Nutrition. 2015;101(5):908-13. doi: 10.3945/ajcn.114.103713. PubMed PMID: WOS:000354075100005.

22. Clark HR, Goyder E, Bissel P, Blank L, Peters J. How do parents' child-feeding behaviours influence child weight? Implications for childhood obesity policy. Journal of Public Health. 2007;29(2):132-41. doi: 10.1093/pubmed/fdm012. PubMed PMID: WOS:000247474600007.

23. Moreau D, Kalaboka S, Choquet M, Annesi-Maesano I. Asthma, obesity, and eating behaviors according to the Diagnostic and Statistical Manual of Mental Disorders IV in a large population-based sample of adolescents. American Journal of Clinical Nutrition. 2009;89(5):1292-8. doi: 10.3945/ajcn.2008.26954.

PubMed PMID: WOS:000265394300004.

24. Arvaniti F, Priftis KN, Papadimitriou A, Yiallouros P, Kapsokefalou M, Anthracopoulos MB, et al. Salty-Snack Eating, Television or Video-Game Viewing, and Asthma Symptoms among 10- to 12-Year-Old Children: The PANACEA Study. Journal of the American Dietetic Association. 2011;111(2):251-7. doi: 10.1016/j.jada.2010.10.051. PubMed PMID: WOS:000287073800012.

25. Wood LG, Garg ML, Gibson PG. A high-fat challenge increases airway inflammation and impairs bronchodilator recovery in asthma. Journal of Allergy and Clinical Immunology. 2011;127(5):1133-40. doi: 10.1016/j.jaci.2011.01.036. PubMed PMID: WOS:000290018600006. 
26. Conn KM, Halterman JS, Fisher SG, Yoos HL, Chin NP, Szilagyi PG. Parental beliefs about medications and medication adherence among urban children with asthma. Ambulatory Pediatrics. 2005;5(5):306-10. doi: 10.1367/a05-004r1.1. PubMed PMID: WOS:000232119000010.

27. De Simoni A, Horne R, Fleming L, Bush A, Griffiths C. What do adolescents with asthma really think about adherence to inhalers? Insights from a qualitative analysis of a UK online forum. Bmj Open. 2017;7(6):12. doi: 10.1136/bmjopen-2016-015245. PubMed PMID: WOS:000406391200138.

28. Horne R, Weinman J. Self-regulation and self-management in asthma: Exploring the role of illness perceptions and treatment beliefs in explaining non-adherence to preventer medication. Psychology \& Health. 2002;17(1):17-32. doi: 10.1080/08870440290001502. PubMed PMID: WOS:000176071800002.

29. Forno E, Lescher R, Strunk R, Weiss S, Fuhlbrigge A, Celedon JC, et al. Decreased response to inhaled steroids in overweight and obese asthmatic children. Journal of Allergy and Clinical Immunology.

2011;127(3):741-9. doi: 10.1016/j.jaci.2010.12.010. PubMed PMID: WOS:000288018400029.

30. King LA, Loss JHM, Wilkenfeld RL, Pagnini DL, Booth ML, Booth SL. Australian GPs' perceptions about child and adolescent overweight and obesity - the Weight of Opinion study. British Journal of General Practice. 2007;57(535):124-9. PubMed PMID: WOS:000245338500009.

31. Shrewsbury VA, King LA, Hattersley LA, Howlett SA, Hardy LL, Baur LA. Adolescent-parent interactions and communication preferences regarding body weight and weight management: a qualitative study. International Journal of Behavioral Nutrition and Physical Activity. 2010;7. doi: 10.1186/1479-5868-716. PubMed PMID: WOS:000275316800001.

32. Alexander GL, Olden HA, Troy T, Miree CA, Joseph CLM. Overweight adolescents and asthma: Revealing motivations and challenges with adolescent-provider communication. Journal of Asthma. 2018;55(3):266-74. doi: 10.1080/02770903.2017.1323921. PubMed PMID: WOS:000426104600006. 33. Nelson PA, Keyworth C, Chisholm A, Pearce CJ, Griffiths CEM, Cordingley L, et al. 'In someone's clinic but not in mine' - clinicians' views of supporting lifestyle behaviour change in patients with psoriasis: a qualitative interview study. British Journal of Dermatology. 2014;171(5):1116-22. doi: 10.1111/bjd.13231. PubMed PMID: WOS:000346600000005.

34. Schalkwijk AAH, Bot SDM, de Vries L, Westerman MJ, Nijpels G, Elders PJM. Perspectives of obese children and their parents on lifestyle behavior change: a qualitative study. International Journal of Behavioral Nutrition and Physical Activity. 2015;12:8. doi: 10.1186/s12966-015-0263-8. PubMed PMID: WOS:000359618900001. 
35. Brown I, Thompson J, Tod A, Jones G. Primary care support for tackling obesity: a qualitative study of the perceptions of obese patients. British Journal of General Practice. 2006;56(530):666-72. PubMed PMID: WOS:000241448100006.

36. National Institute for Health and Care Excellence. Obesity: identification, assessment and management. NICE [Internet]. 2014 November [cited 2018 Jun 5]. Available from:

https://www.nice.org.uk/guidance/cg189/chapter/1-recommendations

Table 1 - Demographic characteristic of participants

\begin{tabular}{|l|l|}
\hline Characteristics & N \\
\hline Job title & 3 \\
Paediatric Respiratory Nurse Specialist & 4 \\
Consultant in Paediatric Respiratory Medicine & 3 \\
Consultant in General Paediatric Medicine & \\
Gender & 3 \\
Male & 7 \\
Female & \\
Age & 2 \\
$30-39$ & 5 \\
$40-49$ & 1 \\
50+ & 2 \\
Unspecified & \\
Frequency of contact with paediatric asthma & \\
patients & 5 \\
Daily & 4 \\
Weekly & 1 \\
Unspecified & \\
Ethnicity & 5 \\
White British & 4 \\
Asian - Indian & \\
Asian - unspecified & 1 \\
\hline
\end{tabular}

